ESAIM: PROCEEDINGS, March 2012, Vol. 35, p. 281-286

Fédération Denis Poisson (Orléans-Tours) et E. Trélat (UPMC), Editors

\title{
CEREBRAL BLOOD FLOW SIMULATIONS IN REALISTIC GEOMETRIES
}

\author{
StÉPhanie SALMON ${ }^{1}$, Soyibou SY ${ }^{2}$ AND MARCEla SZOPOS ${ }^{3}$
}

\begin{abstract}
The aim of this work is to perform the computation of the blood flow in all the cerebral network, obtained from medical images as angiographies. We use free finite elements codes as FreeFEM++. We first test the code on analytical solutions in simplified geometries. Then, we study the influence of boundary conditions on the flow and we finally perform first computations on realistic meshes.

Résumé. L'objectif est ici de simuler l'écoulement sanguin dans tout le réseau cérébral (artériel et veineux) obtenu à partir d'angiographies cérébrales 3D à l'aide de logiciels d'éléments finis libres, comme FreeFEM++. Nous menons d'abord une étude détaillée des résultats sur des solutions analytiques et l'influence des conditions limites à imposer dans des géométries simplifiées avant de travailler sur les maillages réalistes.
\end{abstract}

\section{INTRODUCTION}

This work is part of a larger project whose final objective is to compute virtual cerebral angiography images. In order to do so, we need to reconstruct the total arterial and/or venous cerebral network from medical images, transform the vascular model obtained in a computational mesh and compute the blood flow in all the network. The final step is then to simulate the physical mechanisms of acquisition of the images to compute virtual angiographies (see also [1] for a similar approach).

First of all, we need to reconstruct the cerebral network from medical images obtained by collaborators in several hospital structures (Hôpital Civil de Strasbourg, Hôpital Louis-Pasteur de Colmar). By segmentation techniques and discrete image analysis, we obtain vascular models (see Figure 1) which are blocks of voxels representing the vessels [5] (voxels $=$ volumes elements). These models allow to obtain a surface mesh which has to be pre-processed in order to obtain the volumic mesh suitable for computation. For example, we need to label the inlets and outlets to be able to impose the necessary boundary conditions for the mathematical model. This step from vascular models to computational meshes is realized by an in-house code, called "Cutmesh" that was developed by O. Génevaux [3] and some public open ones as FreeYams [2] to smooth the surface triangulation. Then, the volumic mesh is achieved by a tetrahedral generator (see Figure 2, left panel, for the entire mesh and Figure 2, right panel, for a zoom of a part of this image, which shows the mesh in detail).

\footnotetext{
${ }^{1}$ Laboratoire de Mathématiques EA 4535, Université de Reims, U.F.R. Sciences Exactes et Naturelles, Moulin de la Housse BP 1039, 51687 REIMS cedex 2

${ }^{2}$ Institut de Recherche Mathématique Avancée, UMR 7501 CNRS/Université de Strasbourg, 7, rue René Descartes, F-67084 STRASBOURG Cedex

${ }^{3}$ Institut de Recherche Mathématique Avancée, UMR 7501 CNRS/Université de Strasbourg, 7, rue René Descartes, F-67084 STRASBOURG Cedex
}

(C) EDP Sciences, SMAI 2012 


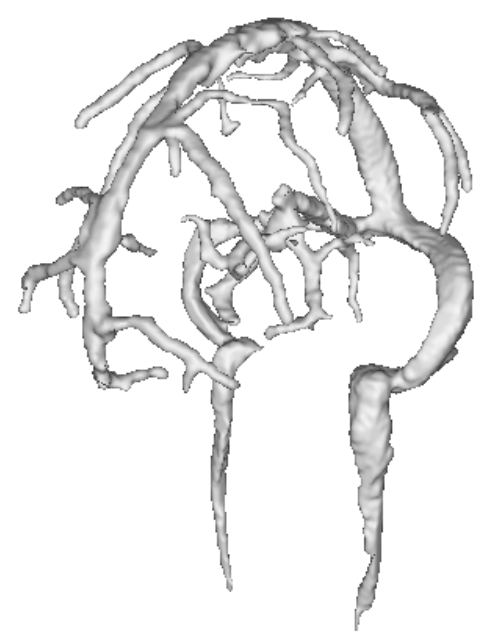

FiguRE 1. Vascular model
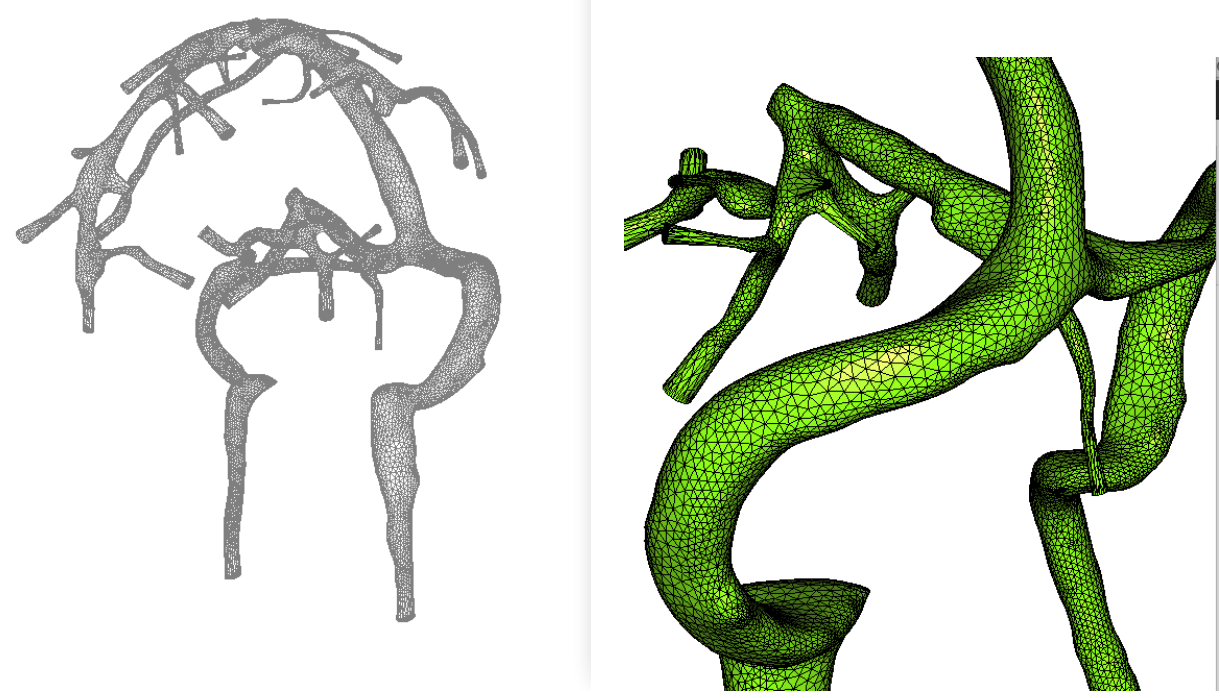

Figure 2. One example of 3D tetrahedral mesh of 163282 elements (left) - Magnified view of the mesh (right)

\section{Modelling AND NUMERICAL EXPERIMENTS}

In a first approximation, the blood is considered as an incompressible Newtonian fluid (see for a similar approach [6] and the references therein) and we neglect the interactions between the vessels walls and the fluid, as the cerebral network is quite constrained in the brain. So we end up with a simple fluid simulation of the 
Navier-Stokes equations.

$$
\begin{cases}\frac{\partial \mathbf{u}}{\partial t}-\nu \Delta \mathbf{u}+\mathbf{u} \cdot \nabla \mathbf{u}+\nabla p=\mathbf{f}, & \text { in } \Omega \\ \operatorname{div}(\mathbf{u})=0, & \text { in } \Omega \\ \mathbf{u}=\mathbf{u}_{0}, & \text { on } \partial \Omega\end{cases}
$$

where $\Omega$ is the fluid domain, $\nu$ the kinematic viscosity, $\mathbf{f}$ the external forces, $\mathbf{u}$ and $p$ stand respectively for the velocity and the pressure of the fluid and $\mathbf{u}_{0}$ denotes the boundary velocity.

We use the free finite element code FreeFem $++{ }^{1}$ for the simulations of the blood flow. First of all, we need to investigate the linear and stationary problem. We choose to work with the Stokes equations in symmetric formulation as the natural condition arising from this formulation is the whole stress tensor as shown in the variational formulation above. The symmetric formulation of the Stokes equations reads as

$$
\begin{cases}-2 \nu \operatorname{div}(\epsilon(\mathbf{u}))+\nabla p=\mathbf{f}, & \text { in } \Omega \\ \operatorname{div}(\mathbf{u})=0, & \text { in } \Omega \\ \mathbf{u}=\mathbf{u}_{0} & \text { on } \partial \Omega\end{cases}
$$

We recall that $\epsilon(\mathbf{u})=\frac{1}{2}\left(\nabla \mathbf{u}+(\nabla \mathbf{u})^{T}\right)$ is the shear stress tensor. We also introduce the stress tensor $\sigma(\mathbf{u}, p)=$ $-p \mathbb{I}_{3}+2 \nu \epsilon(\mathbf{u})$ and the operator $\epsilon(\mathbf{u}): \nabla \mathbf{v}:=\operatorname{tr}\left((\epsilon(\mathbf{u}))^{T} \nabla \mathbf{v}\right)$. To obtain the variational formulation, we multiply the first equation by a function $\mathbf{v} \in W$ and the second one by $q \in M$ and we integrate by parts : find $\mathbf{u} \in V$ and $p \in M$ such that

$$
\begin{aligned}
& 2 \nu \int_{\Omega} \epsilon(\mathbf{u}): \epsilon(\mathbf{v}) \mathrm{d} x-\int_{\Omega} p \operatorname{div} \mathbf{v} \mathrm{d} x+\int_{\partial \Omega}(\sigma \mathbf{n}) \cdot \mathbf{v} \mathrm{d} x=\int_{\Omega} \mathbf{f} \cdot \mathbf{v} \mathrm{d} x, \quad \forall \mathbf{v} \in V \\
& -\int_{\Omega} q \operatorname{div} \mathbf{u} \mathrm{d} x-\varepsilon \int_{\Omega} p q \mathrm{~d} x=0, \quad \forall q \in M
\end{aligned}
$$

Spaces $V$ and $M$ are respectively $V=H_{0}^{1}(\Omega)$ and $M=L^{2}(\Omega)$. The term $-\varepsilon \int_{\Omega} p q \mathrm{~d} x$ is usually added in FreeFem++ to obtain an invertible matrix (it is the so-called penalty method, see for details [4], Section 5.3.3).

One of the key problem is the boundary conditions. For the inlet, we need to impose a periodic Poiseuille flow, which is a first approximation of the pulsatile flow going out from the heart. This condition is a Dirichlet one and is imposed strongly. For the outlet, there are several options. The simpler one, the so-called "do nothing" condition is difficult to use with the symmetric formulation. As an example, we show the result of the bidimensional Poiseuille flow, where the solution is known : the velocity has a parabolic profile and the pressure is linear. We compare both solutions, one imposing on the outlet the same Poiseuille flow as on the inlet and one with the "do nothing" condition. We can see on Figure 3 and Figure 4 that we obtain different solutions. For example, the pressure is no more linear with the "do nothing" condition and what is imposed is only that the pressure is null at the middle of the outlet boundary (see [7] for similar results). Indeed, the "do nothing" condition corresponds to impose the normal component of the stress tensor equal to zero and thus the flow is going out as a jet as physically expected. As we want to mimic the rest of the network (little arteries beyond the image resolution, veins and capillaries), which has to be cut in order to keep a reasonable simulation in terms of time and memory, we have to be aware that the "do nothing" condition is not the appropriate one for our simulations. Indeed, the solution would be to couple the 3D simulation with a $1 \mathrm{D}$ model.

The next step we perform is to validate the results with an analytical solution proposed by Ethier and Steinmann [8]. We consider a cube $\Omega=[0,1]^{3}$ and solve the three-dimensional Stokes problem $(\nu=1)$ with

\footnotetext{
${ }^{1}$ http://www.freefem.org
} 

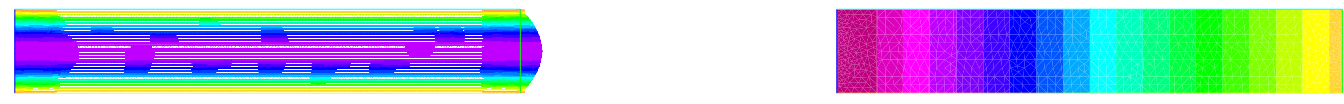

FIGURE 3. Velocity and pressure with a Poiseuille flow strongly imposed at the inlet and the outlet.
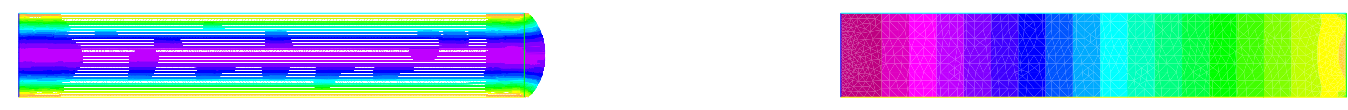

FIGURE 4. Velocity and pressure with a Poiseuille flow strongly imposed at the inlet and the "do nothing" condition at the outlet.

Dirichlet boundary conditions on the velocity using a symmetric variational formulation. Assume the exact solution given by:

$$
\begin{aligned}
u_{1}(x, y, z) & =-a\left(e^{a x} \sin (a y+d z)+e^{a z} \cos (a x+d y)\right) \\
u_{2}(x, y, z) & =-a\left(e^{a y} \sin (a z+d x)+e^{a x} \cos (a y+d z)\right) \\
u_{3}(x, y, z) & =-a\left(e^{a z} \sin (a x+d y)+e^{a y} \cos (a z+d x)\right) \\
p(x, y, z) & =-\frac{a^{2}}{2}\left(e^{2 a x}+e^{2 a y}+e^{2 a z}+2 \sin (a x+d y) \cos (a z+d x) e^{a(y+z)}\right. \\
& +2 \sin (a y+d z) \cos (a x+d y) e^{a(z+x)} \\
& \left.+2 \sin (a z+d x) \cos (a y+d z) e^{a(x+y)}\right)
\end{aligned}
$$

with parameters $a=\pi / 4, d=\pi / 2$ and a source term $f$ given by $\mathbf{f}=-\nu \Delta \mathbf{u}+\nabla p$. The penalty parameter $\varepsilon$ is chosen 1.0e-5. The linear solver is GMRES, and the stop criterion of GMRES is 1.0e -6 .

We have tested two types of elements : first $\mathbb{P}^{1}+$ bubble for the velocity and $\mathbb{P}^{1}$ for the pressure and second the $\mathbb{P}^{2}$ for the velocity and $\mathbb{P}^{1}$ for the pressure. Both of them are theoretically verifying the inf-sup conditions necessary to obtain a well-posed Stokes problem. Nevertheless, results obtained with the $\mathbb{P}^{1}+$ bubble $-\mathbb{P}^{1}$ are not satisfactory : convergence is achieved but pressure stays far from the expected one. Thus, for our simulations we will prefer the $\mathbb{P}^{2}-\mathbb{P}^{1}$ element.

Next, in order to assess the accuracy of the numerical solutions, the graphs related to the velocity's and pressure's error estimations have been studied. In logarithmic scale, we represent the errors $e_{u, 0}=\left\|u_{h}-u_{e x}\right\|_{L^{2}}$ (see Figure 5) and $e_{p, 0}=\left\|p_{h}-p_{e x}\right\|_{L^{2}}$ (see Figure 6) related to the mesh step $h$. The graphs show a convergence order close to the one predicted by the theory (the loss of precision being due to the geometry approximation in $\left.\mathcal{O}\left(h^{2}\right)\right)$. 


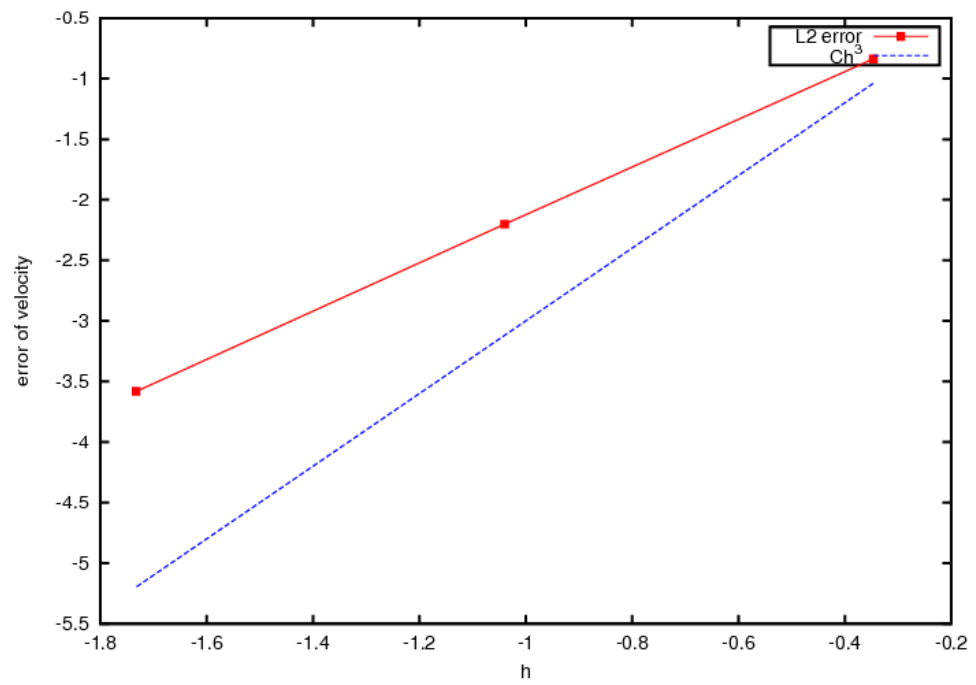

FigURE 5. Error estimation of the velocity.

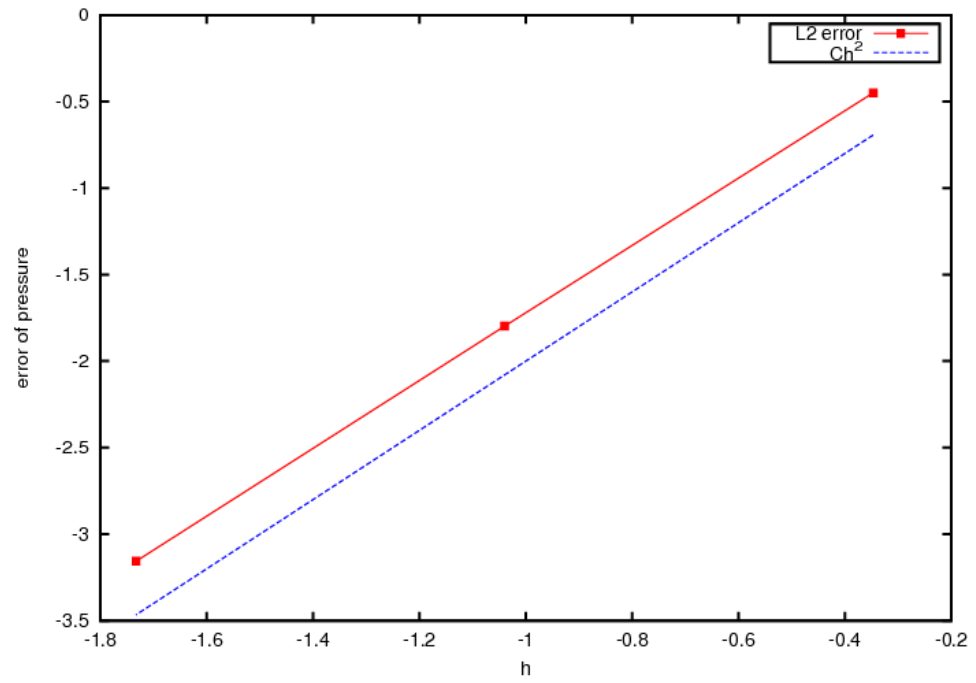

Figure 6. Error estimation of the pressure.

\section{First NUMERICAL RESUltS AND PROSPECTS}

Finally, we compute our first example on a real mesh obtained from medical images, which proves that the whole chain from images to computation is achievable. We begin with a stationary and linear simulation, with FreeFEM++ installed on CLOVIS (computing center of the Region Champagne-Ardenne ${ }^{2}$ ) and with $\mathbb{P}^{1}+$ bubble $-\mathbb{P}^{1}$ element (despite our first numerical results). We impose a Poiseuille flow at the two inlets at the bottom, all the other branches are considered outlets. We plot streamlines on Figure 7 and we can remark that the flow in the middle artery comes mostly for the right branch.

\footnotetext{
$2_{\text {www.romeo2.fr/ }}$
} 

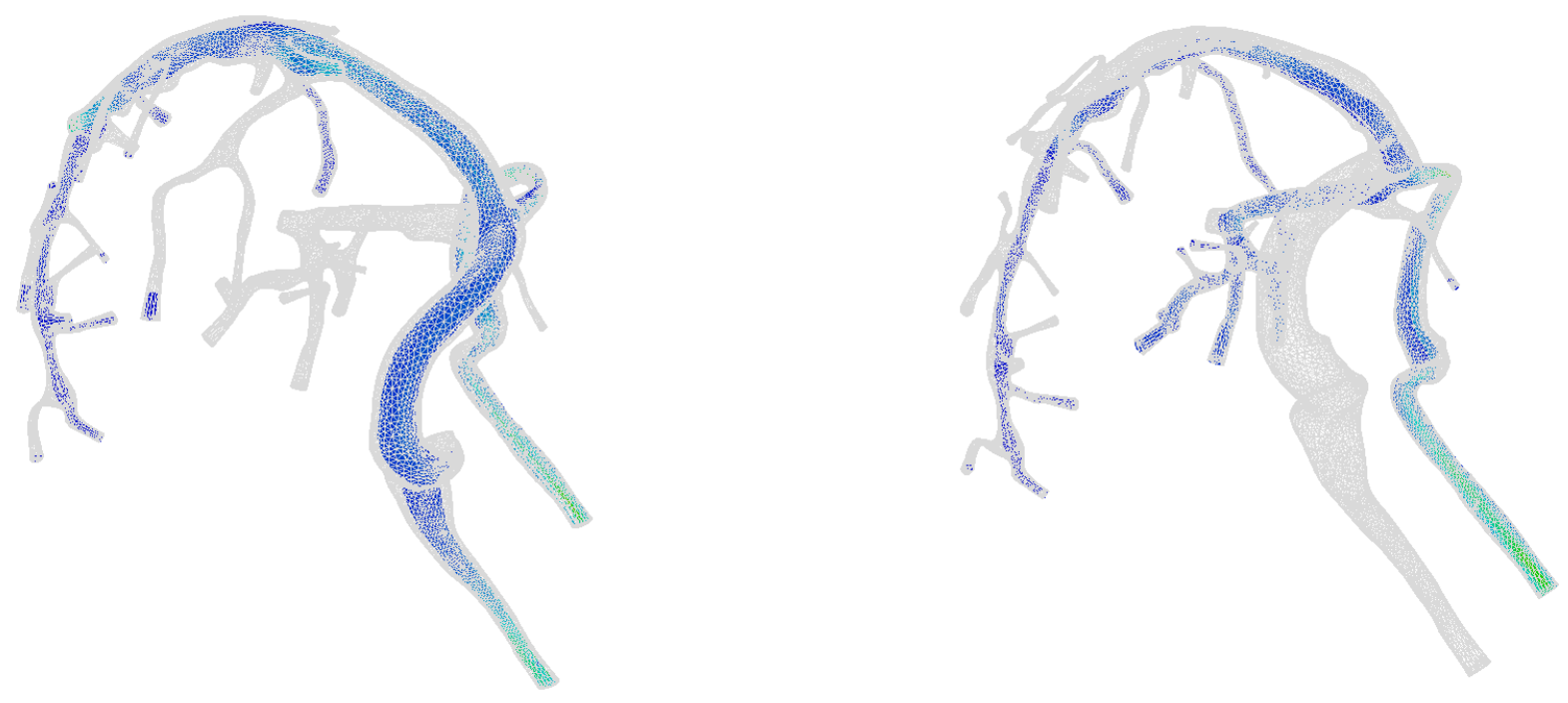

Figure 7. Stationary flow - Streamlines (FreeFrem++ ).

Future work is to perform the simulation correctly, using $\mathbb{P}^{2}-\mathbb{P}^{1}$ element and appropriate boundary conditions as this network is a venous one (so inlets and outlets should be inverted). We are also working on arterial networks and particularly the so-called Willis polygon whose geometry is quite complex.

\section{ACKNOWLEDGEMENT}

The authors want to thank the University of Reims-Champagne-Ardenne for the financial support and the referees for helping us to improve this article.

\section{REFERENCES}

[1] M.D. Ford, G.R. Stuhne, H.N. Nikolov, D.F Habets, S.P. Lownie, D.W. Holdsworth, D.A. Steinman Virtual angiography for visualization and validation of computational models of aneurysm hemodynamics. IEEE Trans Med Imaging 24(12):1586-1592 (2005).

[2] P. Frey, A fully automatic adaptive isotropic surface remeshing procedure. INRIA RT0252 (2001).

[3] Olivier Génevaux, Logiciel "Cutmesh", 2010.

[4] A. Ern, J.-L. Guermond, Éléments finis: théorie, applications, mise en œuvre. (French) [Finite elements: theory, applications, implementation] Mathématiques \& Applications (Berlin) [Mathematics \& Applications], 36. Springer-Verlag, Berlin (2002).

[5] N. Passat, C. Ronse, J. Baruthio, J.-P. Armspach, C. Maillot, Magnetic resonance angiography: From anatomical knowledge modeling to vessel segmentation, Medical Image Analysis, 10(2):259-274 (2006).

[6] A. Quarteroni, L. Formaggia, Mathematical modelling and numerical simulation of the cardiovascular system, Handbook of numerical analysis. Vol. XII:3-127, North-Holland, Amsterdam (2004).

[7] Assia Soualah-Alila, Modélisation mathématique et numérique du poumon humain, PhD Paris XI University (2007).

[8] C. Ethier And D. Steinman, Exact fully 3d Navier-Stokes solutions for benchmarking., Int. J. Numer. Methods Fluids 19 (1994) 369-375. 